\section{Tingimento natural eco-friendly de ripas de bambu com Curcuma longa}

\section{Resumo}

Este estudo apresenta resultados parciais de uma pesquisa que explora as possibilidades de colorir ripas de bambu a partir de técnicas ecologicamente amigáveis. As ripas de Bambusa vulgaris foram utilizadas para realizar processos de tingimento natural usando Curcuma longa como um corante natural. Diferentes tonalidades são obtidas como resultado do processo de tingimento através do uso de 3 tipos de mordentes (sulfato alumínico de potássio, acetato de ferro, e lixivia em solução de cinzas rico em soda cáustica). A evidência empírica demonstra processos de tingimento natural do bambu que podem ser inicialmente consideradas como alternativas sustentáveis que usam técnicas simples, muito pouco poluentes e favoráveis ao meio ambiente.
Pedro Arturo Martínez

Osorio

Doutor em Design

Universidade Estadual Paulista, UNESP, Bauru, Brasil

Professor da Corporación Universitaria del

Caribe, Sincelejo, Colombia

Correio eletrônico:

pedro.martinez@cecar.edu.co

(ㄱ) orcid.org/0000-0002-9024-0918

Google Scholar

Paula Da Cruz Landim

Doutora em Arquitetura e Urbanismo,

Universidade Estadual Paulista, UNESP,

Bauru, Brasi

Correio eletrônico:

paula.cruz-landim@unesp.br

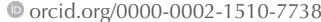

Google Scholar

Tomás Queiroz Ferreira

Barata

Doutor em Engenharia Civil

Universidade Estadual Paulista, UNESP,

Bauru, Brasi

Correio eletrônico: barata@faac.unesp.br

(ㄱ) orcid.org/0000-0002-1573-5590

Google Scholar

Marco Antônio Pereira

Doutor em Agronomia,

Universidade Estadual Paulista, UNESP, Bauru, Brasil

Correio eletrônico: pereira@feb.unesp.br (1) orcid.org/0000-0002-1473-4860

Google Scholar

Recibido: junio 5 de 2018

Aprobado: diciembre 01 de 2019

Palavras chave:

bambu, Curcuma longa,

design sustentável, mordentes, tingimento natural.

Revista KEPES Año 17 No. 21 enero-junio 2020, págs. 437-457 ISSN: 1794-7111(Impreso) ISSN: 2462-8115 (En línea) DOI: $10.17151 /$ kepes.2020.17.21.15 (c) (1) 


\section{Eco-friendly natural dyeing of bamboo slats with Curcuma longa}

\begin{abstract}
This study presents partial results of a research that explores the possibilities of coloring bamboo slats using ecologically friendly techniques. Bambusa vulgaris slats were used to perform natural dyeing processes using Curcuma longa as a natural dye. Different shades are obtained as a result of the dyeing process through the use of 3 types of mordants (potassium aluminum sulfate, iron acetate, and lye solution rich in caustic soda). Empirical evidence demonstrates natural dyeing processes of bamboo that can initially be considered as sustainable alternatives that use simple, very low-polluting and environmentally friendly techniques.
\end{abstract}

Key words:

Bamboo, Curcuma longa, mordents, natural dye, sustainable design. 


\section{Tinte ecológico natural de tiras de bambú con Curcuma longa}

\section{Resumen}

Este estudio presenta resultados parciales de una investigación que explora las posibilidades de teñir tiras de bambú utilizando técnicas ecológicas. Las tiras de Bambusa vulgaris se utilizaron para realizar procesos de teñido natural utilizando Curcuma longa como tinte natural. Se obtienen diferentes tonos como resultado del proceso de tintura mediante el uso de tres tipos de mordentes (sulfato de aluminio y potasio, acetato de hierro y solución de cenizas rica en soda cáustica). La evidencia empírica demuestra procesos de teñido natural de bambú que inicialmente pueden considerarse como alternativas sostenibles que utilizan técnicas simples, limpias y respetuosas con el medio ambiente.
Palabras clave:

bambú, colorante natural, Curcuma longa, diseño sostenible, mordientes. 


\section{Introdução}

Os inúmeros problemas ambientais geraram uma crescente pressão no mercado internacional, que despertou em diferentes cenários o interesse em conhecer e reinterpretar as técnicas tradicionais mais amigáveis com o meio ambiente para o desenvolvimento de sistemas, produtos e serviços sustentáveis, de modo a desenvolver processos naturais substituindo técnicas industriais altamente poluentes.

O design, entre outras disciplinas, se preocupa nesses aspectos e atualmente relaciona conceitos de sustentabilidade sempre tomando em conta o ecologicamente necessário, para desenvolver "sistemas-produto" que sejam "social e culturalmente apreciáveis" (Manzini \& Vezzolli, 2002, p. 20).

Nesse sentido o acervo inerente às técnicas tradicionais relacionadas ao artesanato, cada vez mais, gera o interesse de diferentes disciplinas entre as quais o design acerca-se para gerar diálogos significativos na busca de referências para inovação e como forma de ressignificação dos valores locais, devido a que são fortemente enraizadas em sua relação com a cultura e território (Organización de las Naciones Unidas -ONU, 1992).

Por outra parte a redução do impacto ambiental nos processos de produção encontrou na biotecnologia uma fonte de conhecimento e recursos a serem aplicados em diferentes contextos. O conhecimento e as técnicas milenares sobre o uso de plantas, animais e minerais são utilizadas como ponto de partida para gerar novos desenvolvimentos e inovações em torno dos problemas, necessidades e exigências da sociedade contemporânea (Carvalho \& Santos, 2015).

Recentemente, a indústria de tintas começou gradualmente a ter interesse em retomar o uso de corantes naturais em substituição de corantes químicos, o 
que levou novamente a exploração das possibilidades de tingir com diferentes plantas, devido às suas potencialidades, tais como antialérgicos, repelentes de insetos, antimicrobianos, retardadores de combustão, proteção UV, etc. (Rather et al., 2016; Hurtado \& Arroyo, 2016; Kumaresan, 2016).

A revived interest in the use of natural dyes in textile and food coloration has been growing since last few decades and there is an urgent need for availability of natural dye yielding plants for fulfilling the purpose. Recently discovered properties of natural dyes such as insect repellent, deodorizing, flame retardant, UV protection, fluorescence, and antimicrobial activity, besides being biocompatible, biodegradable, renewable, and non-toxic have revolutionized all industrial sectors especially textile industry for producing more appealing and highly functional value-added textiles. (Rather et al., 2016)

Assim que, nos últimos anos é cada vez mais valorado o uso de corantes naturais devido a suas propriedades e grande variedade de aplicações nos processos de inovação no desenvolvimento de produtos com alto valor agregado.

Por outra parte, sobre o bambu que atualmente é uma das espécies vegetais com maior perspectiva, com uma importância crescente no mundo (Pereira \& Beraldo, 2008), existe um crescente interesse na experimentação de suas potencialidades desde a perspectiva do design. Inúmeros de produtos e materiais são pesquisados com a finalidade de atingir as necessidades contemporâneas sempre relacionando o bambu com aspectos da sustentabilidade como material versátil, económico e resistente.

Apresentam-se neste artigo os resultados parciais de uma pesquisa sobre as potencialidades de tingimento natural das ripas de bambu, observando técnicas tradicionais indígenas, como ponto de partida para a reinterpretação de técnicas aplicáveis ao desenvolvimento de produtos de design sustentável com bambu. 


\section{Revisão de literatura}

Em relação às pesquisas recentes sobre processos de tingimento usando corantes naturais, é importante notar que este é um campo de pesquisa que gradualmente ganha popularidade devido às preocupações com a redução de impactos ambientais e à diversificação de produtos ecologicamente corretos que dão solução para as crescentes necessidades do mercado.

Embora seja verdade que os corantes naturais foram aplicados desde tempos antigos com múltiplos usos, o interesse pelo uso diminuiu após a descoberta e difusão de corantes sintéticos (Mirjalili, Nazarpoor, \& Karimi, 2011). Atualmente, além de preocupações ambientais, cada vez mais pesquisas estão sendo feitas em corantes naturais, relacionados a aspectos de segurança e efeitos sobre a saúde, principalmente devido a doenças causadas por corantes sintéticos, especialmente em bebês e crianças (Mirjalili et al., 2011).

Shahid, Shahid-ul-Islam e Mohammad (2013), realizaram uma revisão em pesquisas recentes sobre os diferentes usos de corantes naturais, e encontraram aplicações tais como: acabamento têxtil, coloração alimentar, tingimento de couro, polpa, plásticos, adição de cores a cosméticos, corantes capilares, impressão de cor para produtos farmacêuticos, entre outros. Os autores observaram a necessidade de compreender e melhorar muitos aspectos técnicos no uso desses recursos naturais para um uso eficiente na indústria (Shahid et al., 2013).

Nesse sentido, manter a força e a resistência das cores é uma das preocupações das pesquisas desenvolvidas com corantes naturais. Mohini, Tejashree y Vijay, (2018) analisam o tingimento de Thespesia populnea (T. populnea) em diferentes têxteis, observando os efeitos da lavagem com agua, sabonete, luz solar, efeito do alume, e efeito de sulfato cúprico; Shahid et al. (2017) exploram 
a influência do tratamento de radiação gama em tingimento têxtil com Tagetes erecta L.; Hurtado e Arroyo (2016) medem os resultados de cores após o uso de processos de pré-mordentes com alume na coloração de fibras naturais com diferentes plantas. No mesmo sentido Rather et al. (2016), Kumaresan (2016), Prabhu e Teli (2014), Saravanan e Chandramohan (2011), respectivamente, avaliam as possibilidades de tingimento usando Adhatoda vasica, Spathodea campanulata, Tamarindus indica L., y Ficus religiosa L. como corantes naturais, analisam os efeitos do uso de diferentes mordentes e suas combinações. Entretanto, Mayusoh (2015) pesquisa o design de padrões através de processos de tingimento natural.

Sobre os processos de tingimento usando Curcuma longa, pode se observar que essa planta é muito referenciada em pesquisas desenvolvidas sobre corantes naturais, a qual é reconhecida pelas propriedades antimicrobianas da curcumina que é o corante natural obtido da planta (Shahid et al., 2013).

A Curcuma longa, conhecida na Colômbia nas comunidades indígenas Zenú pelo nome "batatilla", é usada pelos indígenas para o tingimento da fibra de Gynerium sagittatum na elaboração de artesanato trançado, obtendo colorações amarelas e marrons.

A técnica de tingimento dos indígenas Zenú usando Curcuma longa é processo simples no qual se tingem por cocção as fibras de Gynerium sagittatum. Primeiro as raízes de cúrcuma são descascadas e, em seguida, liquefeitas em água para coar a mistura. Depois se executa o cozimento, durante o processo é adicionado um pouco de sal que age como mordente.

Também Nogueira, Gomes e Moreira (2017), quem explicam a obtenção de corantes naturais em São João de Côrtes, Alcântara, Maranhão, Brasil; entre os processos que são resgatados no estudo aparecem plantas como Curcuma longa, 
chamada no Brasil açafrão, Bixa orellana L., chamada urucum, Rhizophora mangle, entre outras. O estudo de Nogueira et al. (2017), foi orientado a compreender as possibilidades que o design focado na sustentabilidade tem nos conhecimentos ancestrais como acervo e referencia constante para a inovação.

Por outro lado, os processos de tingimento de madeira, embora menos frequentes, despertaram recentemente o interesse dos pesquisadores, tentando responder às necessidades de um mercado crescente de madeira exótica, difícil de obter e de crescimento prolongado, razão pela qual os processos de tingimento se tornaram uma solução alternativa para o problema. Pesquisas como as de Guo, Liu e Li, (2011), Guan, Zhu e Song, (2016) e Zhang, Yu e Wu, (2015), entre outros, exploram as possibilidades de tingir madeira de reflorestamento de rápido crescimento, para alcançar os tons e aparência de madeiras tropicais exóticas.

Em relação aos processos de tingimento com bambu, é possível identificar vários estudos que analisam o potencial de absorção de pigmentos do bambu como material para o tratamento de gases e líquidos poluentes, principalmente pela produção industrial de têxteis. Chan, Cheung, Mckay, (2008), Chan, Cheung, Allen e Mckay (2009), Mui (2010), Peng et al. (2012), Wang (2012), entre outros, exploram as capacidades de absorção de pigmentos do carbono e do carbono ativado do bambu, como alternativa para mitigar a contaminação por pigmentos sintéticos.

Em outro sentido, pesquisas como a de Zhang, Xu, \& Chen (2014), analisam as possibilidades de tingir com pigmentos vegetais, para o desenvolvimento de eco-têxteis com base em fibras de bambu. As propriedades de tingimento das fibras viscosas de bambu foram estudadas neste trabalho, usando Gardenia yellow y sodium copper chlorophyll. Identificam fatores importantes que 
influenciam o processo, como o uso de mordentes, o $\mathrm{pH}$, bem como a temperatura, o tempo e a dosagem do mordente (Zhang et al., 2014).

Entretanto, Ya et al. (2016) pesquisam a influência da mudança de cor da tintura básica usando "Basic Brown G", sobre: módulo de ruptura (MOR), módulo de elasticidade (MOE), resistência ao corte (carga paralela), velocidade de inchaço de espessura e taxa de absorção de fibra, em compósitos de bambu feitos com Phyllostachys pubescens (Ya et al., 2016). Este estudo é uma referência importante para novas pesquisas sobre materiais compósitos baseados em fibras de bambu e sobre o comportamento das fibras de bambu nos processos de tingimento.

Assim, as pesquisas recentes relacionadas com o tema demonstram um campo de pesquisa em expansão que inclui explorações sobre uso de corantes naturais, sua aplicação e as potencialidades do bambu como alternativa para mitigar problemas de contaminação com pigmentos, e como opção sustentável ao uso de madeiras exóticas.

\section{Materiais e métodos}

O material utilizado para este estudo corresponde a ripas de Bambusa vulgaris coletadas in natura na cidade de Bauru, SP, Brasil. O pigmento natural utilizado foi Curcuma longa, adquirido em uma apresentação em pó, comprado em um mercado local.

O processamento das tiras de bambu foi realizado com referência aos processos tradicionais de tratamento descritos por Pereira e Beraldo (2008). 12 ripas de bambu foram preparadas com dimensões $10 \mathrm{~mm} \times 100 \mathrm{~mm}$, com um peso total de $21 \mathrm{~g}$ (figura 1). 


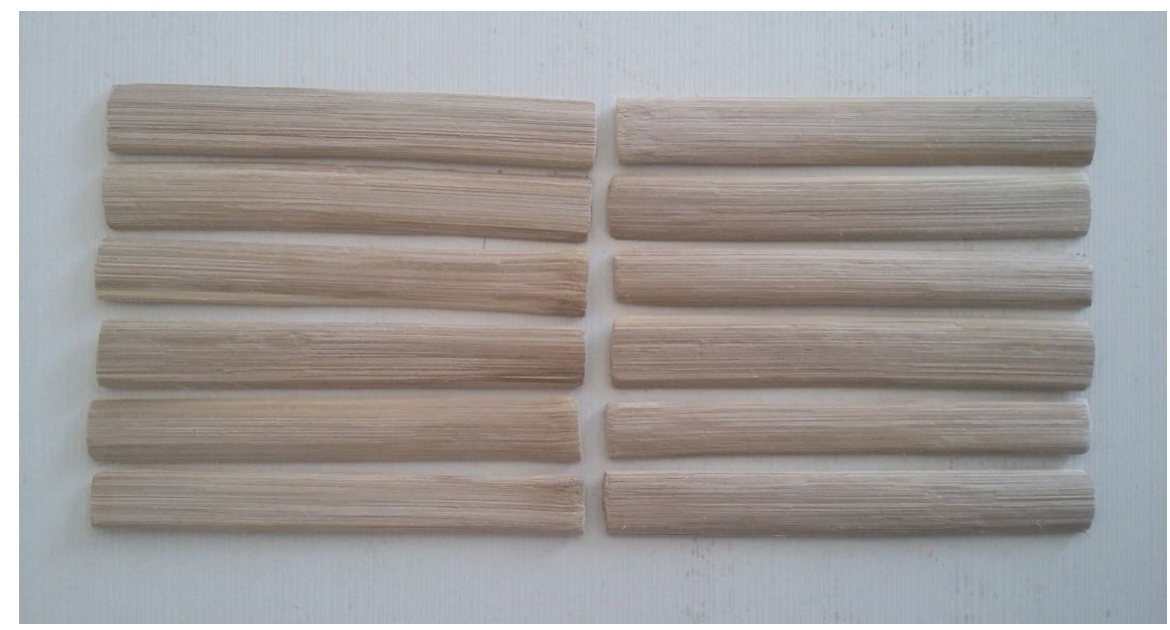

Figura 1. Ripas de Bambusa vulgaris antes do processo de tingimento. Fonte: os autores.

O método de tingimento natural utilizado foi desenvolvido observando como referência o processo de tingimento dos indígenas Zenú da Colômbia, que consiste no tingimento por cocção das fibras de Gynerium sagittatum. Procedeuse a reinterpretar a técnica aplicando-a ao tingimento de ripas de Bambusa vulgaris. É importante anotar que o processo de tingimento dos indígenas Zenú com "batatilla" (Curcuma longa) no inclui o processo de fixação com mordentes após da cocção, devido a que nestes eles usam sal como mordente durante o processo, portanto aqui se exploram outras possibilidades a partir dos processos observados com os indígenas.

O processo de fixação das cores nas peças de bambu foi realizado utilizando 3 tipos de mordentes naturais: sulfato alumínico de potássio $\left(\mathrm{SO}_{4}\right)_{2} \mathrm{ALK} 12 \mathrm{H}_{2} \mathrm{O}$ (alúmen de potássio), acetato de ferro $\left.\mathrm{Fe}\{\mathrm{CH} 3 \mathrm{COO})_{2}\right) 4 \mathrm{H}_{2} \mathrm{O}$, e lixivia em 
solução de cinzas rico em soda cáustica $\mathrm{NaOH}$ (decoada), tomando como referencia os métodos de Lopes (1998) e Kawakami (2017).

Desenvolveram-se 3 etapas, preparação do tingimento, tingimento por cocção, e fixação das cores com mordentes, as quais são descritas a seguir:

A etapa 1, preparação do tingimento, na qual definiram-se as soluções para o tingimento e para a fixação das cores com mordentes. Para a preparação da solução tintora conformada pela mistura de água com Curcuma longa, a quantidade de corante natural definiu-se a partir do peso do material a ser tingido numa proporção 2:1. Neste caso, para $21 \mathrm{~g}$ de ripas de Bambusa vulgaris foram utilizados $10,5 \mathrm{~g}$ de corante (50\% em peso). A quantidade de água para a realização da mistura definiu-se a partir do peso do corante a ser usado numa proporção 1:20. Neste caso foram usados $2000 \mathrm{ml}$ de água para fazer uma solução com una concentração de $5 \% \mathrm{~m} / \mathrm{V}$.

Para a preparação dos mordentes foram usados $100 \mathrm{ml}$ de solução de água com mordente por cada 5,25 g de ripas (4 unidades), os quais foram distribuídos em recipientes plásticos separados assim: alume de potássio numa concentração de $10 \% \mathrm{~m} / \mathrm{V}$; acetato de ferro numa concentração de $29 \% \mathrm{~m} / \mathrm{V}$; e solução de cinzas numa concentração de $20 \% \mathrm{~m} / \mathrm{V}$.

A etapa 2, tingimento por cocção, que consistiu no processo de cocção da solução tintora num recipiente de alumínio onde foram mergulhadas simultaneamente as ripas de Bambusa vulgaris. A solução foi deixada até ferver num fogão convencional, logo depois foi abaixada a temperatura deixando ao fogo por 15 minutos a mais. Posteriormente, são retiradas as ripas da mistura para sua lavagem na água fria (figura 2). 


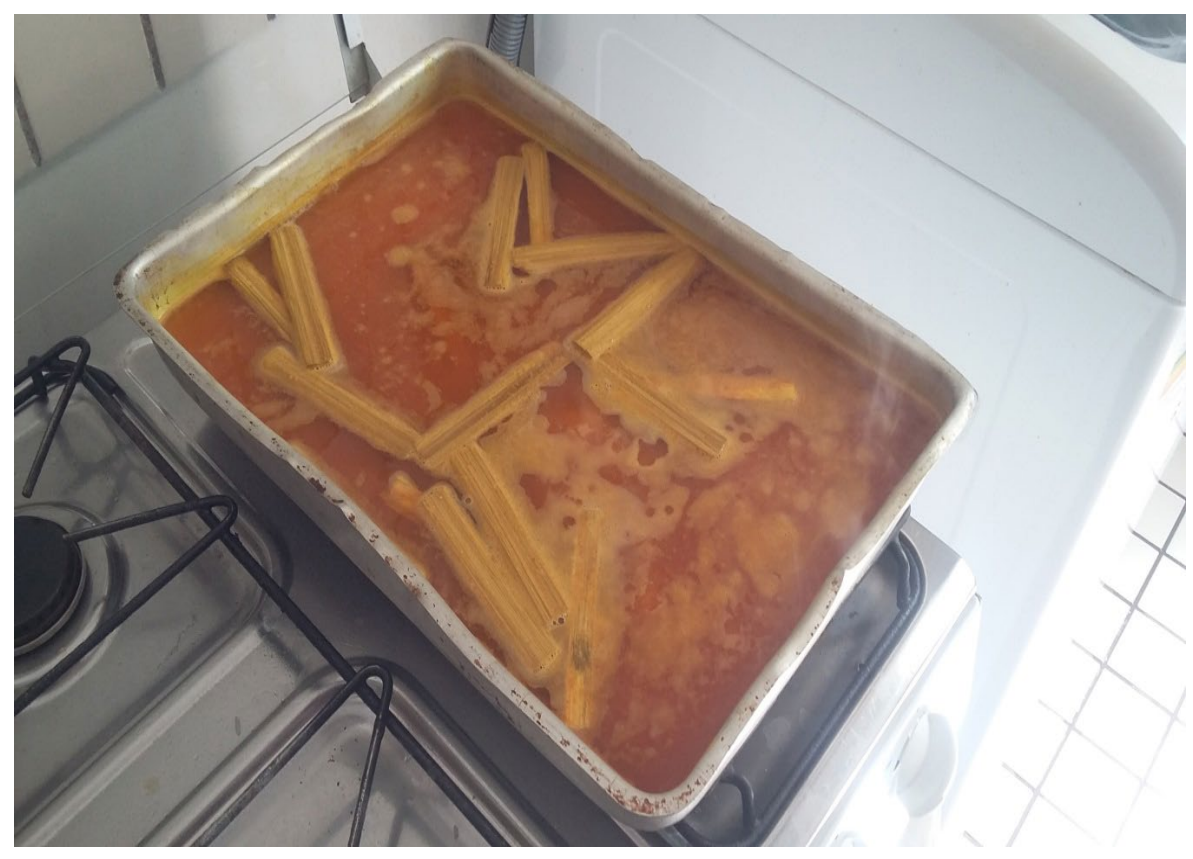

Figura 2. Processo de tingimento por cocção das ripas de Bambusa vulgaris. Fonte: foto dos autores.

A etapa 3, fixação das cores com mordentes, consistiu na imersão das peças na solução mordente. Foram introduzidas 4 ripas $(5,25 \mathrm{~g})$ em recipientes separados de cada mordente durante 15 minutos (figura 3). Finalmente as ripas são retiradas e lavadas com água fria, deixadas a secar e comparadas às transformações das cores. 


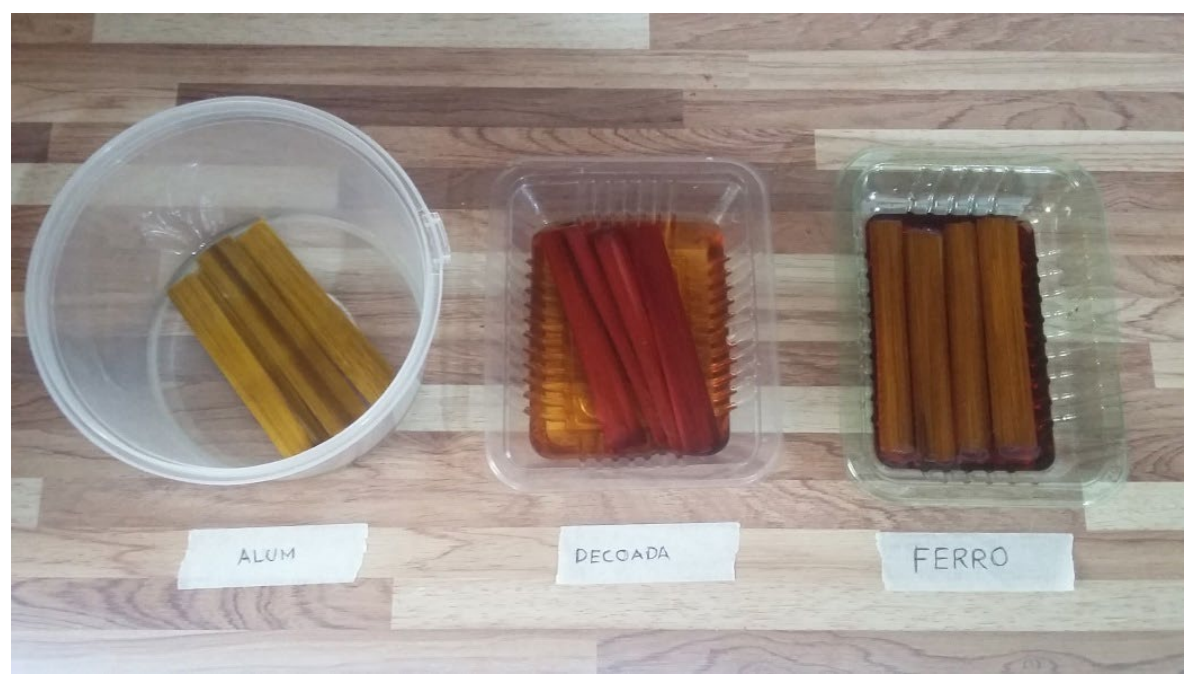

Figura 3. Processo de fixação das cores nas ripas de Bambusa vulgaris. Fonte: foto dos autores.

\section{Resultados e discussão}

Inicialmente o primeiro resultado obtido do processo de tingimento das ripas de Bambusa vulgaris com Curcuma longa, foi a mudança das cores depois do processo de cocção. Retiradas às peças e lavadas com água fria, observou-se que a totalidade das ripas adquiriu uma cor amarela bastante consistente. 


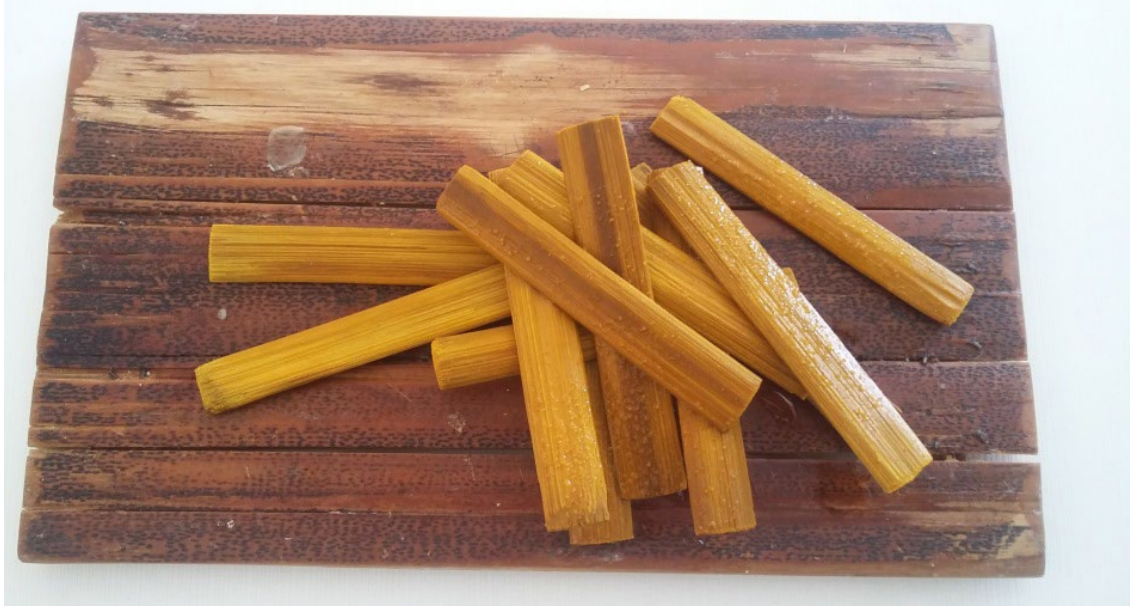

Figura 4. Ripas de Bambusa vulgaris despois do processo de cocção em solução aquosa de Curcuma longa. Fonte: foto dos autores.

Posteriormente a partir do processo de fixação das cores com mordentes, mudanças na coloração foram observadas nas ripas. Após da imersão das ripas na solução de mordente, três tons diferentes foram obtidos como produto do processo de tingimento natural com Curcuma longa (figura 5).

As ripas no mordente de alúmen de potássio apresentaram uma cor amarela ligeiramente mais leve do que as peças antes de serem submersas na solução. As ripas que foram submersas na solução de cinzas ou decoada, apresentaram uma forte transformação na cor, adquirindo a cor vermelha intensa. As ripas que foram introduzidas na solução de acetato de ferro apresentaram uma transformação moderada da cor, tornando-se um pouco mais escura do que as peças antes de serem submersas. 


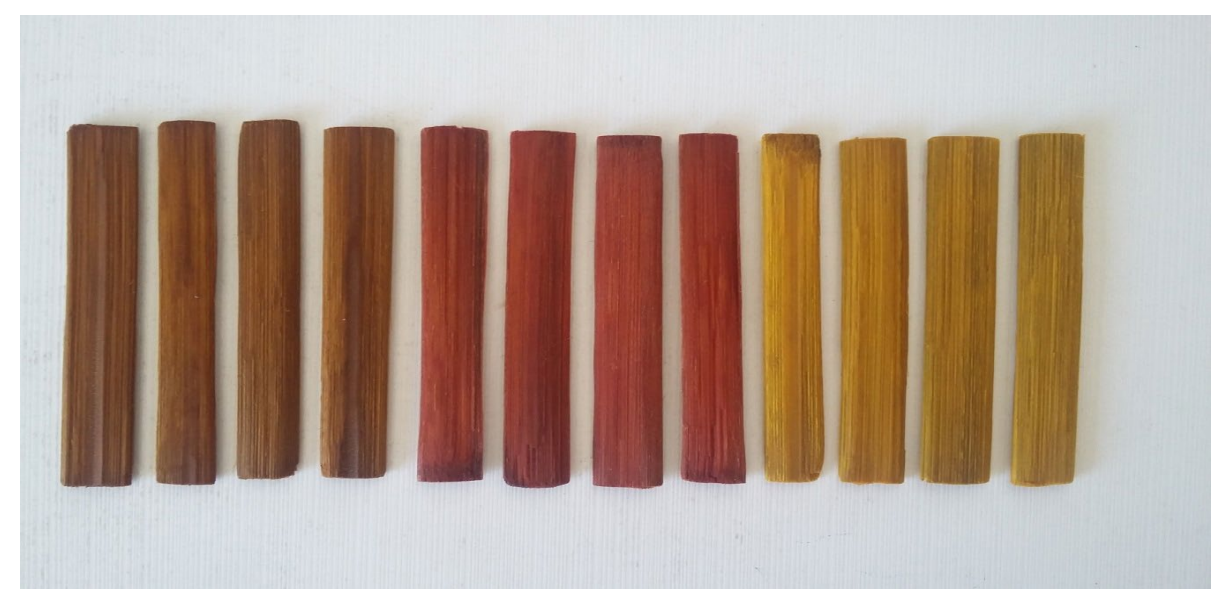

Figura 5. Ripas de Bambusa vulgaris depois de serem sometidas ao processo de tingimento. Fonte: foto dos autores.

Embora este estudo tenha sido considerado apenas como variável para medir a coloração os diferentes tipos de mordentes, alguns desses resultados preliminares podem ser assimilados com o descrito por Zhang et al. (2014), quando eles explicam seus resultados em relação à obtenção dos índices mais altos de profundidade em cores com os processos pós-mordente, como os realizados neste estudo, porque mudanças moderadas e drásticas também foram observadas na coloração das peças tingidas após o mordente ser aplicado; melhorando o processo e possibilitando a aquisição de um amplo espectro de cores, como explicado por Shahid et al. (2013).

É importante em um estudo posterior ampliar o foco do analise para comparar o tingimento usando Curcuma longa, com os resultados encontrados por Mohini et al. (2018), os quais observaram os efeitos da lavagem com agua, sabonete, 
luz solar, efeito do alume, e sulfato cúprico no tingimento com T. populnea. Nesse sentido, também é interessante realizar uma pesquisa que proponha uma comparação de resultados em processos de coloração natural usando pré e pós-mordentes como os processos descritos por Hurtado e Arroyo (2016), aplicados agora à tintura de ripas de bambu, o que permitiria explorar outras possibilidades e estabelecer processos mais eficientes de tingimento.

Em relação aos processos para alterar a coloração natural das madeiras a partir de processos de tingimento conforme descrito por Guo et al. (2011), Guan et al. (2016) e Zhang et al. (2015), também é possível demonstrar processos de tingimento natural com ripas de bambu, que podem ser utilizadas no desenvolvimento de produtos de design sustentável de bambu, com uma grande variedade de tonalidades, agregando valor, usando uma tecnologia muito simples, aplicável ao desenvolvimento de vários produtos, em quase todos os contextos e com muito pouco consumo de energia.

Sobre as desvantagens e danos no material do qual falam Zhang et al. (2015) no processo de tingimento de madeira, pode-se afirmar que neste estudo em que as ripas foram sujeitas a um processo de tingimento por cozimento, o material aparentemente não foi afetado durante o processo. É interessante expandir a pesquisa para determinar também a afetação das propriedades físicas do material, tomando como referência o estudo desenvolvido por Ya et al. (2016).

Também é interessante gerar uma pesquisa em relação às propriedades antimicrobianas da curcumina, que se referenciadas por Shahid et al. (2013). Uma vez que os resultados obtidos abrem a possibilidade de explorar possíveis produtos com as propriedades do composto presente em Curcuma longa, para o desenvolvimento de produtos de design sustentável com valor agregado aplicável a necessidades particulares de ambientes que exigem condições específicas de assepsia. 


\section{Conclusão}

Como conclusões preliminares deste estudo, as evidências empíricas em relação aos processos de tintura natural do bambu podem ser inicialmente consideradas, usando técnicas simples, muito pouco poluentes e favoráveis ao meio ambiente.

Os resultados preliminares apresentados estimulam um campo de pesquisa em relação ao uso de corantes naturais para o tingimento de madeiras alternativas, especialmente em termos de tingimento de bambu. A necessidade de desenvolver estudos que considerem variáveis múltiplas para entender muito mais os aspectos relacionados no processo e para poder transferir os resultados de uma maneira eficiente para a indústria.

Como os aspectos para melhorar o processo de pesquisa são propostos: controle da temperatura variável durante o cozimento; controlar a concentração de corante natural e mordente para determinar a influência de diferentes concentrações nos resultados; explorar as possibilidades de reutilização das soluções utilizadas e a água para a lavagem dos materiais, a fim de analisar seus efeitos no cálculo do ciclo de vida dos possíveis produtos a serem desenvolvidos.

Finalmente, é muito importante expandir os objetivos do estudo e analisar além das propriedades de coloração do material vegetal utilizado, fazer uma indagação sobre o efeito do processo nas propriedades físicas das ripas de bambu após a aplicação do tingimento. 


\section{Referências}

Carvalho, C. and Santos, G. (2015). Global Communities, Biotechnology and Sustainable Design - Natural / Bio Dyes in Textiles. Procedia Manufacturing, 3, 6557-6564. doi: 10.1016/j.promfg.2015.07.956

Chan, L.S., Cheung, W.H., Allen, S.J., and Mckay, G. (2009). Separation of aciddyes mixture by bamboo derived active carbon. Separation and Purification Technology, 67, 166-172. doi: 10.1016/j.seppur.2009.03.020

Chan, L.S., Cheung, W.H., and Mckay, G. (2008). Adsorption of acid dyes by bamboo derived activated carbon. Desalination, 218, 304-312. doi: 10.1016/j.desal.2007.02.026

Guan, X., Zhu, Y., and Song, W. (2016). Application of RBF neural network improved by peak density function in intelligent color matching of wood dyeing. Chaos, Solitons and Fractals, 89, 485-490. doi: http://dx.doi. org/10.1016/j.chaos.2016.02.015

Guo, M., Liu, X., and Li, Z. (2011). Studies on the Dye-uptake of Fraxinus Mandshurica Veneer with Reactive Dyes. Applied Mechanics and Materials, 44-47, 2288-2292. doi: 10.4028/www.scientific.net/AMM.44-47.2288

Hurtado, E.A. y Arroyo, G. (2016). Teñido de fibras naturales con colorantes naturales. Jóvenes en la ciencia, Revista de Divulgación Científica, 2 (1), 1134-1138.

Kawakami, H. (2017). Tintura vegetal. Guia para treinamento minicurso $13^{\circ}$ Colóquio de moda, 10 edição internacional. UNESP. Bauru: documento sem publicar. Universidade Estadual Paulista Júlio de Mesquita Filho, Bauru, Brasil.

Kumaresan, M. (2016). Application of eco-friendly natural dye obtained from Spathodea campanulata on silk using combination of mordants. 
Management of Environmental Quality: An International Journal, 27(1), 1521. doi: 10.1108/MEQ-04-2015-0061

Lopes, E. (1998). Corantes naturais da flora brasileira. Guia pratica de tingimento com plantas. Curitiba: Optagraf Editora e Gráfica Ltda.

Manzini, E., and Vezzoli, C. (2002). O desenvolvimento de produtos sustentáveis. Os requisitos ambientais dos produtos industriais. São Paulo, Brasil: Editora da Universidade de São Paulo.

Mayusoh, C. (2015). The art of designing, fabric pattern by tie-dyeing with natural dyes. Procedia - Social and Behavioral Sciences, 197, 1472-1480. doi: 10.1016/j.sbspro.2015.07.097

Mirjalili, M., Nazarpoor, K., and Karimi, L. (2011). Eco-friendly dyeing of wool using natural dye from weld as co-partner with synthetic dye. Journal of Cleaner Production, 19, 1045-1051. doi: 10.1016/j.jclepro.2011.02.001

Mohini, K., Tejashree, L., and Vijay, N. (2018). Dataset on analysis of dyeing property of natural dye from Thespesia populnea bark on different fabrics. Data in Brief, 16 (February), 401-410 doi: https://doi.org/10.1016/j. dib.2017.11.063

Mui, E.L.K., Cheung, W.H., Valix, M., and Mckay, G. (2010). Dye adsorption onto char from bamboo. Journal of Hazardous Materials, 177, 1001-1005. doi: 10.1016/j.jhazmat.2010.01.018

Nogueira, C., Gomes, R. e Moreira, D. (2017). Os corantes naturais extraídos em São João de Côrtes, Alcântara, Maranhão: uma abordagem orientada para o design sustentável. Mix Sustentável, Florianópolis, 3(4), 93-107. doi: https://doi.org/10.29183/2447-3073.MIX2017.v3.n4.93-107

Organización de las Naciones Unidas, ONU. (1992). Declaração do Rio sobre ambiente e desenvolvimento. Conferência das Nações Unidas sobre o 
Meio ambiente e o Desenvolvimento (CNUMAD). Rio de Janeiro, Brasil. Disponível em: http://www.onu.org.br/rio20/img/2012/01/rio92.pdf

Peng, L., Zainab, M., Ismael, W., Songhu, Y., Man, T., Kun, W., and Jianguo, B. (2012). Adsorption of dyes from aqueous solutions by microwave modified bamboo charcoal. Chemical Engineering Journal, 195-196, 339-346. doi:10.1016/j.jhazmat.2010.10.012

Pereira, M. e Beraldo, A. (2008). Bambu em corpo e alma. Bauru, SP, Brasil: Canal 6.

Prabhu, K.H., and Teli, M.D. (2014). Eco-dyeing using Tamarindus indica L. seed coat tannin as a natural mordant for textiles with antibacterial activity. Journal of Saudi Chemical Society, 18, 864-872. doi: http://dx.doi. org/10.1016/j.jscs.2011.10.014

Rather, L.J., Shahid-ul-Islam, Mohd, S., Mohd, N.B., Mohd, S., Mohd, A.K., and Faqeer, M. (2016). Ecological dyeing of Woolen yarn with Adhatoda vasica natural dye in the presence of biomordants as an alternative copartner to metal mordants. Journal of Environmental Chemical Engineering, 4, 3041 3049. doi: http://dx.doi.org/10.1016/j.jece.2016.06.019

Saravanan, P., and Chandramohan, G. (2011). Dyeing of Silk with Ecofriendly Natural Dye obtained from Barks of Ficus Religiosa L. Universal Journal of Environmental Research and Technology, 1(3), 268-273. Disponível em: https://pdfs.semanticscholar.org/606d/ ed8aff00afe03b457aec64c27dffe3a8e927.pdf

Shahid A., Tahsin, G., Muhammad, A., Fazal-ur-Rehman, Muhammad, S., Iram, H., and Naeem, I. (2017). Appraisal of marigold flower based lutein as natural colourant for textile dyeing under the influence of gamma radiations. Radiation Physics and Chemistry, 130, 35-39. doi: http://dx.doi. org/10.1016/j.radphyschem.2016.07.010 
Shahid, M., Shahid-ul-Islam, and Mohammad, F. (2013). Recent advancements in natural dye applications: a review. Journal of Cleaner Production, 53, 310-331. doi: http://dx.doi.org/10.1016/j.jclepro.2013.03.031

Wang, L. (2012). Application of activated carbon derived from 'waste' bamboo culms for the adsorption of azo disperse dye: Kinetic, equilibrium and thermodynamic studies. Journal of Environmental Management, 102, 7987. doi: 10.1016/j.jenvman.2012.02.019

Ya, H., He, M., Zhu, R.X., Zhang, Y.H., Yu, Y.L., and Yu, W.J. (2016). Influence of dyeing treatment on the performance of bamboo-based fibre composites. Journal of Tropical Forest Science, 28(2), 112-120. Disponível em: https:// www.jstor.org/stable/43799214

Zhang, J., Xu, M., and Chen, Y. (2014). Dyeing of Bamboo Viscose Fiber with Gardenia Yellow and Sodium Copper Chlorophyll. Applied Mechanics and Materials, 670-671, 305-308. doi: 10.4028/www.scientific.net/AMM.670671.305

Zhang, Y., Yu, Z., and Wu, X. (2015). Comparison among Parameters Affecting Vertical Vacuum Dyeing of Populus cathayana Rehd. Forest Products Journal, 65 (3/4), 123-128. doi: 10.13073/FPJ-D-14-00064

Como citar: Martínez, P.A., Landim, P.C., Queiroz, T e Pereira, M.A. (2020). Tingimento natural eco-friendly de ripas de bambu com Curcuma longa. Revista KEPES, 17 (21), 437-457. DOI: 10.17151/kepes.2020.17.21.15 